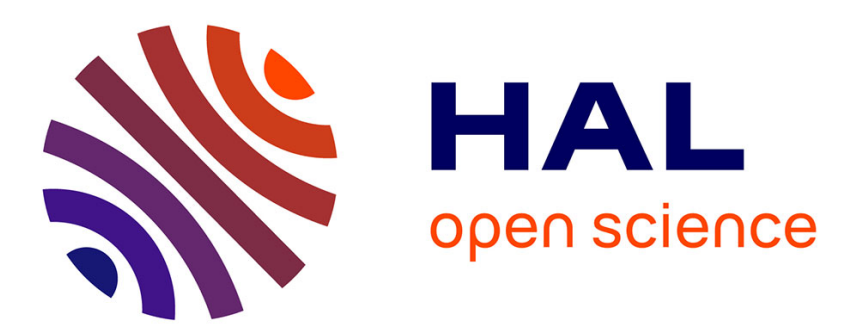

\title{
Coherent combining of mid-infrared difference frequency generators
}

\author{
Alice Odier, Rodwane Chtouki, Pierre Bourdon, Jean-Michel Melkonian, \\ Laurent Lombard, Michel Lefebvre, Anne Durécu
}

\section{To cite this version:}

Alice Odier, Rodwane Chtouki, Pierre Bourdon, Jean-Michel Melkonian, Laurent Lombard, et al.. Coherent combining of mid-infrared difference frequency generators. Optics Letters, 2019, 44 (3), pp.566-569. 10.1364/OL.44.000566 . hal-02317548

\section{HAL Id: hal-02317548 \\ https://hal.science/hal-02317548}

Submitted on 16 Oct 2019

HAL is a multi-disciplinary open access archive for the deposit and dissemination of scientific research documents, whether they are published or not. The documents may come from teaching and research institutions in France or abroad, or from public or private research centers.
L'archive ouverte pluridisciplinaire HAL, est destinée au dépôt et à la diffusion de documents scientifiques de niveau recherche, publiés ou non, émanant des établissements d'enseignement et de recherche français ou étrangers, des laboratoires publics ou privés. 


\title{
Coherent combining of mid-infrared difference frequency generators
}

\author{
Alice Odier ${ }^{1}$, Rodwane Chtouki ${ }^{1, *}$, Pierre Bourdon ${ }^{1}$, Jean-Michel \\ Melkonian $^{1}$, Laurent Lombard ${ }^{1}$, Michel LefebVRe ${ }^{1}$, Anne DuréCu ${ }^{1}$ \\ ${ }^{1}$ ONERA - The French Aerospace Lab, BP 80100, 91123 Palaiseau cedex, France \\ *Corresponding author: rodwane.chtouki@onera.fr
}

Received XX Month XXXX; revised XX Month, XXXX; accepted XX Month XXXX; posted XX Month XXXX (Doc. ID XXXXX); published XX Month XXXX

\begin{abstract}
We report what is, to the best of our knowledge, the first experimental demonstration of coherent combining of two mid-infrared difference frequency generators by active phase control in continuous-wave regime. Using the phase relation that is inherent to the nonlinear process, we are able to phase-lock and combine the idler waves by the sole phase control of one of the pump waves. This control is done by an all fiber electro-optic modulator. Combining is achieved with an excellent efficiency with a residual phase error of $\lambda / 28$. (c) 2019 Optical Society of America
\end{abstract}

OCIS codes: (140.3298) Lasers beam combining; (190.5040) Phase conjugation; (190.4223) Nonlinear wave mixing.

Coherent beam combining (CBC) through active phase control is a well-known solution to overcome limitations to laser power scaling such as thermal effects or damage threshold for solid-state lasers, or nonlinear effects for fiber lasers [1].

Nonlinear frequency converters such as parametric generators used in gas sensing [2-4] could benefit from CBC power scaling, as it would improve the detection range or the sensitivity of the instrument. CBC has been used in conjunction with frequency converters, but only to increase the power of the pump beam, for instance in the case of second harmonic generation (SHG) $[5,6]$. The same idea has been used for optical parametric amplification (OPA) with incoherent combination [7]. However, the inherent limit due to the use of a single nonlinear crystal with limited damage threshold [8] remains. To overcome this limitation, we proposed an indirect phase control approach to combine multiple frequency converters [9], increasing the delivered power without exceeding the damage threshold limit, as the conversion is distributed among multiple nonlinear crystals. Another interest of this indirect phase control technique is the capability to use fast standard all-fiber $1-\mu \mathrm{m}$ or $1.5-\mu \mathrm{m}$ wavelength electro-optic phase modulator (EOM) rather than having to resort to phase modulators operating at much higher wavelengths, which are often less performing or even not commercially available.

Using this approach, we previously reported the experimental demonstration of $\mathrm{CBC}$ by active phase control of continuous wave SHG, achieving the combination of two 532-nm waves from a 1064-nm fundamental wave in the case of perfect phase matching in birefringent lithium triborate (LBO) crystals [10]. The CBC of CW SHG from a $1.55 \mu \mathrm{m}$ wave has also been demonstrated experimentally in the case of quasi-phase matching using periodically poled lithium niobate (PPLN). In both these experiments, phase control operated on the fundamental wave using a standard all-fiber EOM with high bandwidth, and CBC was very efficient with a low residual phase error of $\lambda / 30$ [11].

In this paper, we extend the demonstration to higher wavelength in the mid-infrared range, investigating the combination of difference frequency generators (DFG) emitting at 3.4- $\mu \mathrm{m}$. While SHG is a two-wave degenerate process, we are dealing here with a more complex process involving three non-degenerate waves.

We experimentally demonstrate that CBC with active phase control can nevertheless be implemented using a similar indirect phase control approach as in the case of SHG i.e. with the pump wave phase controlled using standard all-fiber EOM at $1.064 \mu \mathrm{m}$. We observe efficient coherent combining of the idler waves at 3.4$\mu \mathrm{m}$. These results are a first step toward experimental proof of CBC of mid-infrared optical parametric oscillators (OPO) which has already been suggested theoretically [12], to achieve tunable high power mid-infrared sources.

We use the following notations to express the electric field for the pump wave (p), the idler wave (i) and the signal wave (s) with respective angular frequencies $\omega_{p}, \omega_{i}$ and $\omega_{s}$. To simplify this proof of concept, we limit our analysis to continuous wave monochromatic plane waves and we assume the beams to be linearly polarized, collinear, and that we are dealing with a single three-wave-mixing process. We then use scalar fields in the equations. The electric field corresponding to each nonlinearly coupled wave can be written as:

$$
\widetilde{E}_{m}(z, t)=A_{m}(z) e^{j k_{m} z} e^{-j \omega_{m} t}+c . c
$$


with $m=s, i$ or $p$ and $A_{m}$ is the complex amplitude of the angular frequency $\omega_{m}$.

If we assume that the pump wave is not depleted when passing through the crystal, which is true on the first millimeters of the nonlinear crystal where parametric amplification is still weak, the coupled-amplitude equations describing difference frequency generation are [13]:

$$
\left\{\begin{array}{l}
\frac{d A_{\mathrm{s}}(z)}{d z}=\frac{8 \pi j \omega_{\mathrm{s}}^{2} d_{e f f}}{k_{\mathrm{s}} c^{2}} A_{\mathrm{p}} A_{\mathrm{i}}^{*}(\mathrm{z}) e^{j \Delta k z} \\
\frac{d A_{\mathrm{i}}(z)}{d z}=\frac{8 \pi j \omega_{\mathrm{i}}^{2} d_{e f f}}{k_{\mathrm{i}} c^{2}} A_{\mathrm{p}} A_{\mathrm{s}}^{*}(\mathrm{z}) e^{j \Delta k z}
\end{array}\right.
$$

where $\Delta k=k_{\mathrm{p}}-k_{\mathrm{s}}-k_{\mathrm{i}}$

In the case of perfect phase matching (i.e. $\Delta k=0$ ), we differentiate the second equation of (2) with respect to $\mathrm{z}$ and introduce the complex conjugate of the first equation in (2) to eliminate the $\frac{d A_{s}^{*}}{d z}$ term.

We obtain the following equation:

$$
\frac{d^{2} A_{\mathrm{i}}(\mathrm{z})}{d z^{2}}=\kappa_{\mathrm{s}}^{*} \kappa_{i}\left|A_{\mathrm{p}}\right|^{2} A_{\mathrm{i}}(z)
$$

with:

$$
\kappa_{m}=\frac{8 \pi j \omega_{m}^{2} d_{e f f}}{k_{m} c^{2}}
$$

The idler wave being not seeded, we can consider that $A_{i}(0)=$ 0 . The initial value for the signal wave amplitude is noted $A_{s}(0)$. The solutions become:

$$
\left\{\begin{array}{c}
A_{\mathrm{s}}(\mathrm{z})=A_{\mathrm{s}}(0) \cosh \left(\sqrt{\kappa_{\mathrm{s}} \kappa_{\mathrm{i}}^{*}\left|A_{\mathrm{p}}\right|^{2}} \mathrm{z}\right) \\
A_{\mathrm{i}}(\mathrm{z})=\frac{\kappa_{\mathrm{i}} A_{\mathrm{p}}(0)}{\sqrt{\kappa_{\mathrm{s}} \kappa_{\mathrm{i}}^{*}\left|A_{\mathrm{p}}\right|^{2}}} A_{\mathrm{s}}^{*}(0) \sinh \left(\sqrt{\kappa_{\mathrm{s}} \kappa_{\mathrm{i}}^{*}\left|A_{\mathrm{p}}\right|^{2} z}\right)
\end{array}\right.
$$

Writing down the complex field amplitudes using their phase and modulus parts $A_{m}(z)=a_{m}(z) e^{j \varphi_{m}(z)}$, one can verify that the signal field $A_{s}(z)$ retains its initial phase and is simply amplified by the interaction, while the generated idler wave $A_{i}(z)$ has a phase that depends both on that of the pump wave and on that of the signal wave. Noticing from equation (4) that $\kappa_{\mathrm{i}}=\left|\kappa_{\mathrm{i}}\right| e^{j \pi / 2}$, we obtain the following relation:

$$
\varphi_{\mathrm{i}}=\varphi_{\mathrm{p}}-\varphi_{\mathrm{s}}+\frac{\pi}{2}
$$

Similarly, in the QPM case, high conversion efficiency can be achieved in a perfect periodically poled crystal. The relation between phases of the pump, signal and idler waves can be obtained propagating the waves in the crystal domain by domain and step by step in a same way done for SHG $[10,14]$ :

$$
\varphi_{\mathrm{i}}=\varphi_{\mathrm{p}}-\varphi_{\mathrm{s}}
$$

For higher power levels, depletion of the pump wave can become significant and the propagation equations are solved using Jacobi elliptic functions [15]. However, even in this case of strong depletion, equation (5) can be solved step-by-step or domain by domain for QPM, considering there's no pump depletion over one thin slice of the crystal. The initial phase of the signal is thus preserved and seeded step-by-step during propagation, even in long crystals. Consequently, the phase relation "propagates" stepby-step. Periodical poling in QPM crystals compensates for the $\pi$ phase-offset generated between the three coupled waves after propagation through one domain. Thus, the phase relation should remain valid even in the strong depletion regime.

Note that this approximation can be done as long as the gain in one domain or in one thin slice of the medium is weak. This will be the case in continuous wave or nanosecond regimes, but may not be true in picosecond or femtosecond regimes. There is another condition for CBC operation: the combined idler waves must share the same wavelength. To fulfill this condition, it is required to seed the same $1553 \mathrm{~nm}$ signal into both nonlinear crystals. The error signal used here for frequency tagging comes from the idler interference signal following the equation [16]:

$$
S_{e r r}=R_{P D} \sqrt{P_{i 1}} \sqrt{P_{i 2}} \sin \left(\varphi_{i 1}-\varphi_{i 2}\right) J_{1}\left(\beta_{i 2}\right)
$$

where $R_{P D}$ is the photodetectors responsivity, $P_{i}$ the power of each idler wave, $J$ represents a Bessel function of the first kind and $\beta_{i 2}$ is the phase modulation amplitude. Idler phases are linked to the pump and signal ones by relations previously exposed.

An experimental set-up was built to demonstrate such indirect phase control and to validate the theoretical principle presented before. The setup is based on two PPLN crystals pumped by 1.064- $\mu \mathrm{m}$ Yb:fiber sources. The signal seed is provided by 1553 nm Er:fiber sources. Given the wavelength of pump waves, standard high bandwidth all-fiber electro-optic phase modulators operating at the pump wavelength can be used.

A schematic of the experimental set-up for DFG combining is presented in Figure 1.

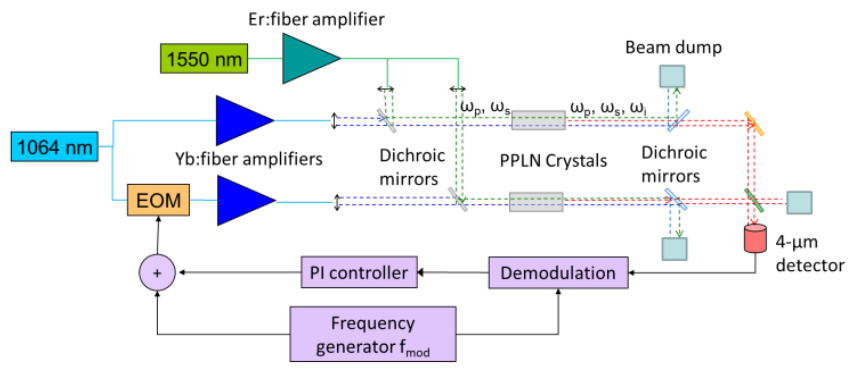

Fig. 1. Schematic of the experimental set-up for demonstrating coherent combining of two 3.4- $\mu \mathrm{m}$ idler beams generated through DFG in PPLN crystals. Coherent combining is achieved by active phasecontrol of one of the pump waves. [17]

Two $1.06 \mu \mathrm{m}$ Yb:fiber amplifiers are seeded by a master oscillator (MO), a laser module (Orion laser module from RIO) at $1.06 \mu \mathrm{m}$. These two amplifiers are homemade ytterbium-doped fiber amplifiers (the same we used in our previous work [11]), each of them capable of delivering up to $15 \mathrm{~W}$ power.

A standard fiber-coupled electro-optic modulator (Photline) controls the phase of one of the $1.06 \mu \mathrm{m}$ fiber amplifiers. This EOM provides both the phase modulation for frequency-tagging and the proper phase shifts to compensate for the phase fluctuations.

To generate the signal seed at $1553 \mathrm{~nm}$, a laser diode is used as a master oscillator. Amplification of this oscillator is achieved using a commercial erbium-doped fiber amplifier (Keopsys, $10 \mathrm{~W}$ ) split in two to seed both DFG channels with a common signal wavelength.

Signal and pump beams are collimated to the same diameter and overlapped. Half-wave plates are used to align the linear polarizations of the 4 beams along the $z$-axis of each PPLN crystal. 
These PPLN DFG crystals were provided by Covesion and their length are $L=20 \mathrm{~mm}$. With a 1064-nm pump wavelength and a seed signal at 1553-nm, the quasi-phase matching in the PPLN crystal is obtained with a poling period of $30.49 \mu \mathrm{m}$ at an operating temperature of $60^{\circ} \mathrm{C}$ for both crystals, generating an idler wave at $3.4 \mu \mathrm{m}$. The pump beams are focused down to a $100 \mu \mathrm{m} 1 / e^{2}$ radius waist in the PPLN crystals.

At the output of each nonlinear crystal, a dichroic mirror separates the residual signal and pump beams from the 3.4- $\mu \mathrm{m}$ idler beam. The two idler beams are then overlapped using a $50 / 50$ pellicle beam splitter. A coated germanium window filters off the residual signal and pump photons, so that the infrared detector used for phase control only receives the $3.4 \mu \mathrm{m}$ beams interference pattern. This photodetector is an InSb cooled detector. The far-field interference signal at $3.4 \mu \mathrm{m}$ provides the feedback needed to close the phase-locking loop.

Frequency tagging operates at $56 \mathrm{kHz}$, and phase error signal generation is performed using a commercial lock-in amplifier. This error signal is driven to 0 by a proportional integral (PI) controller, used to send the proper command in terms of phase shift on the EOM.

The goal of these preliminary experiments is to demonstrate the feasibility of indirect phase control, not to establish records in terms of DFG efficiency, the DFG efficiency is indeed low, with only a few milliwatts at $3.4 \mu \mathrm{m}$, as the lasers are continuous-wave operating. DFG efficiency maximization would have required some enhancement: higher pump power or longer crystals [18].

Before performing indirect phase control, we first verified the phase relation between the pump, signal and idler waves. To measure phase differences, pump, signal and idler beams are overlapped to create interferences pattern. An In-phase and Quadrature (I/Q) detection is implemented, placing photodetectors at different places of the interferences pattern for the signals to be shifted in phase quadrature.

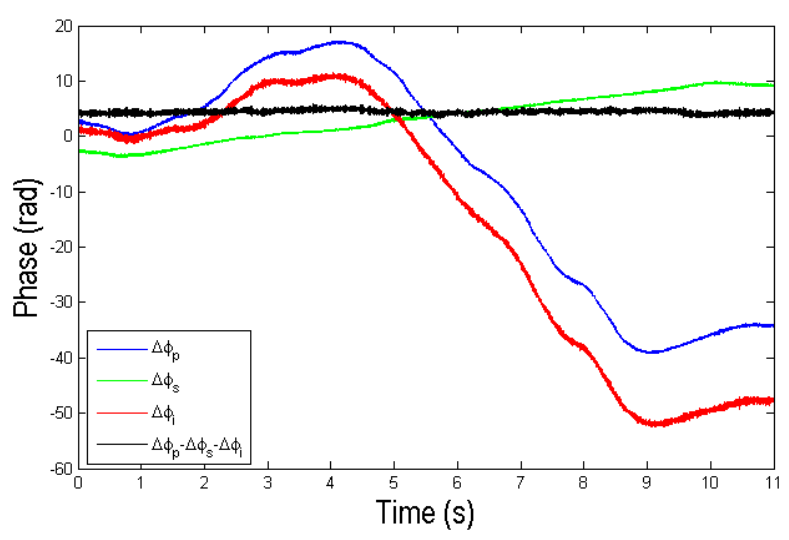

Fig. 2. Phase measurements when the control loop is open.

Figure 2 presents an example of free phase fluctuation measurements (i.e. when the phase-locking control loop is inactive) for each wave. We measured the phase difference between each wave at the output of the two nonlinear crystals $\Delta \varphi=\varphi_{2}-\varphi_{1}$ for the pump, signal and idler. The difference $\Delta \varphi_{p}-\Delta \varphi_{s}-\Delta \varphi_{i}$ is also plotted. According to equation (7), this difference should be equal to zero, but here it is not the case due to optical path differences between the two channels. This constitutes the experimental proof of the theoretically expected relation between the phase of pump, signal and idler waves.

As DFG is a three-wave nonlinear process, one could expect the need to actively control simultaneously the phases of two of the coupled waves to achieve efficient CBC. In practice, it is not the case as active phase control of the sole pump wave is sufficient to efficiently combine the idler waves. The reason for that is explained thereafter.

In terms of phase offsets of the different waves, using the indices 1 and 2 for each optical channel, the phase relation for each channel can be written $\varphi_{i 1}=\varphi_{p 1}-\varphi_{s 1}+c_{1}$ and $\varphi_{i 2}=\varphi_{p 2}-$ $\varphi_{s 2}+c_{2}$ respectively. The constants $c_{1}$ and $c_{2}$ are introduced as a general expression of the constant phase offset between the 3 waves, due to potential optical path difference that can appear as both optical channels are not perfectly equidistant in the experimental configuration.

In order to obtain constructive interference between the two idler beams, their phase offsets must be equal: $\varphi_{i 1}=\varphi_{i 2}$. This condition corresponds to: $\varphi_{p 1}-\varphi_{s 1}+c_{1}=\varphi_{p 2}-\varphi_{s 2}+c_{2}$. This relation can be achieved by controlling the phase of the pump beam on the second channel and only this phase, in order to maintain in real time:

$$
\varphi_{p 2}=\varphi_{p 1}-\varphi_{s 1}+\varphi_{s 2}-\Delta c
$$

Proper value of the second channel pump beam will be found automatically by the feedback loop controlling it, in order to compensate for the slow thermal fluctuations of phase of both signal channels and of the first channel pump

As a consequence, in order to coherently combine the idler waves, there is no need to control both pump and signal phases. Idler CBC can be achieved through controlling the phase of the sole pump wavelength on the second channel, as long as the signal waves on both channels are seeded by the same wavelength from a single-frequency laser source.

As the fixed phase relation has been demonstrated both theoretically and in practice, indirect active phase control can be used to perform coherent beam combining. Figure 3 presents the intensity measured in the center of the idler interference pattern by an InSb cooled detector, when the control loop is open and then closed.

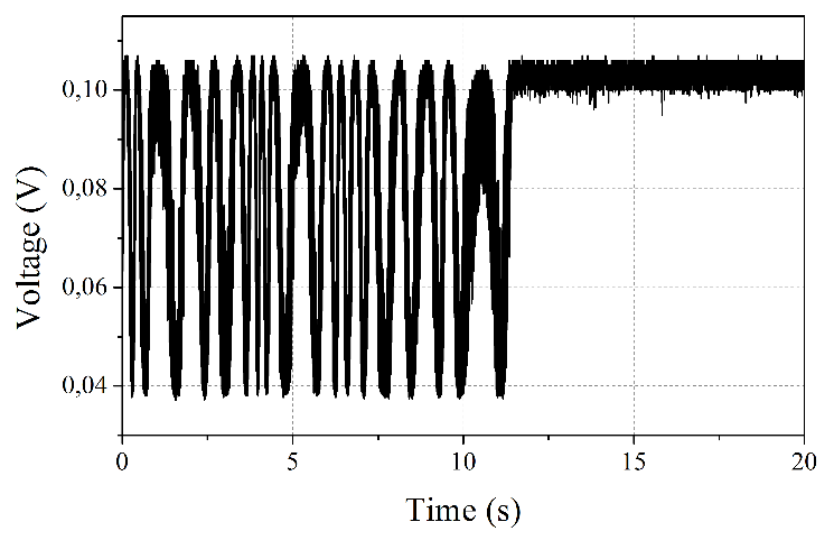

Fig. 3. Time evolution of the interference signals of the idler beams when the phase control loop is open and then closed. 
As long as the phase-control loop is open, the idler interference signal fluctuates freely between destructive and constructive states. When the feedback-loop is closed, the idler interference signal is locked on a constructive interference and the signal measured by the photodiode is kept near its maximum value.

The time-averaged combining efficiency is excellent: the residual phase error is $\lambda / 28 \mathrm{rms}$ for the idler wave. This experiment is the proof that CBC of CW DFG using indirect phase control is possible.

Since we are able to phase-lock the idler wave interferences in a constructive state, one can wonder how the phase difference of the pump and signal wave evolve. Figure 4 presents an example of phase fluctuations of the pump and the signal waves when the phase control loop is active.

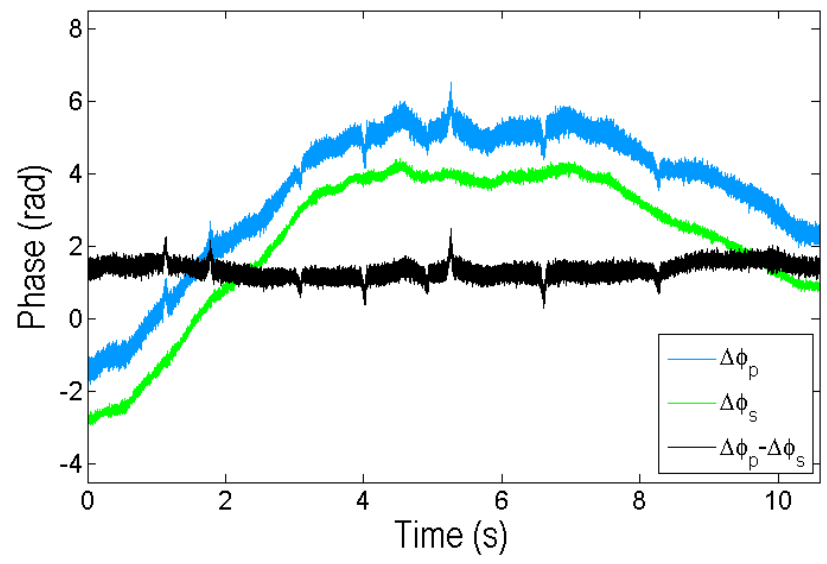

Fig. 4. Phase measurements when the control loop is closed.

Due to the low level of efficiency of the DFG process and the very low power of the idler beams, we could not use fast mid-infrared detectors as they did not have the required sensitivity. We had to use slower InSb cooled detection with a higher sensitivity, and a slower lock-in amplifier that induced a strong electronic noise. As we did not have more than $2 \mathrm{InSb}$ detectors, we also used one of them for simultaneous I/Q detection for phase measurement and phase-locking detection. This explains the lower quality of the phase measurement, as the I/Q detection accuracy suffered a lot from the electronic noise induced by sharing one detector with the phase-locking feedback loop.

Consequently, the phase measurement of the idler wave was irrelevant and hence not plotted in figure 4. This electronic noise also slowed down the voltage shift used to bring back the EOM voltage to the center of its operating range when it reaches its boundaries. One consequence is a much slower shift visible on figure 4 on the blue and black graphs: the spikes visible on these curves correspond to the longer than normal voltage shifts.

In figure 4 , the signal phase difference is varying freely. The pump phase is controlled to compensate those fluctuations according to equation (9). Thus, it will be impossible, by indirect control of one single wave, to phase-lock simultaneously the idler and the signal waves. We demonstrated previously, in the case of SHG CBC [11], that simultaneous phase-locking of both the fundamental and second harmonic waves was feasible, but it will be impossible in the non-degenerate case of DFG CBC. The difference $\Delta \phi_{p}-\Delta \phi_{s}$ is also plotted. We know from Figure 3 that $\Delta \phi_{i}=0$ since we were able to lock the interferences in a constructive state. Therefore, the black line in Figure 4 should be equal to $-\Delta c=c_{1}-c_{2}$ which is related to the real optical path differences of the experimental configuration. The black curve is slowly varying around a constant average value, following the thermal fluctuations of the optical path differences.

In this paper, the first experimental demonstration of coherent combining of difference frequency generators by active phase control using all-fiber components is presented and investigated.

Direct measurements of the residual phase difference between the pump beams and between the idler beams experimentally confirm the well-known fixed relation existing between the pump, signal and idler phases.

Efficient combining of a pair of difference frequency generators is achieved with a residual phase error lower than $\lambda / 28 \mathrm{rms}$ in this DFG CBC experiment. In a three wave non-degenerated process, we were able to phase lock the idler beams using only one control loop.

This important result opens the way to expand this technique of indirect phase control to other nonlinear devices with higher parametric gain and efficiency, such as CW OPOs.

Funding. Direction Générale de l'Armement.

Acknowledgements. The authors are grateful to Antoine Godard for critical reading of the manuscript and valuable suggestions.

\section{References}

1. S. J. Augst, T. Y. Fan, and A. Sanchez, Opt. Lett. 29, 474-476 (2004)

2. Kulp, T., Bisson, S., Bambha, R. et al. Appl Phys B (2002) 75: 317.

3. P. Weibring, H. Edner, and S. Svanberg, Appl. Opt. 42, 3583-3594 (2003)

4. H. Riris, K. Numata, S. Li, S. Wu, A. Ramanathan, M. Dawsey, J. Mao, R. Kawa, and J. B. Abshire, Appl. Opt. 51, 8296-8305 (2012)

5. S.-W. Chiow, T. Kovachy, J. M. Hogan and M. A. Kasevich, Opt. Lett. 37 (18), 3861-3863 (2012)

6. L. R. Taylor, Y. Feng and D. Bonaccini Calia, Opt. Express 18 (8), 8540-8555 (2010)

7. G. Mennerat, B. Trophème, and B. Boulanger, Opt. Lett. 38, 3319-3321 (2013)

8. Y. Q. Zheng, H. Y. Zhu, L. X. Huang, H. B. Chen, Y. M. Duan, R. B. Su, C. H. Huang, Y. Wei, J. Zhuang and G. Zhang, Laser Phys. 20 (4), 756-760 (2010)

9. A. Durécu, G. Canat, J. Le Gouët, L. Lombard, and P. Bourdon, in CLEO: 2014, OSA Technical Digest (online) (Optical Society of America, 2014), paper JTh2A.19.

10. P. Bourdon, A. Durécu, G. Canat, J. Le Gouët, D. Goular, L. Lombard, Proc. SPIE 9344, 93441S (2015);

11. Odier, A., Durécu, A., Melkonian, J. M., Lombard, L., Lefebvre, M., and Bourdon, P. , Optics Letters, 42 (16), 3201-3204 (2017).

12. Liwen Feng, Xiaojun Wang, and Weiwei Ke, J. Opt. Soc. Am. B 34, 991-997 (2017)

13. Arlee V. Smith, Crystal Nonlinear Optics: With SNLO Examples, ASPhotonics, 2018

14. M. M. Fejer, G. A. Magel, D. H. Jundt and R. L. Byer, IEEE Journal of Quantum Electronics, vol. 28, no. 11, pp. 2631-2654, Nov. 1992.

15. R. Baumgartner and R. Byer, IEEE Journal of Quantum Electronics, vol. 15, no. 6, pp. 432-444, June 1979.

16. Shay, Thomas. (2007). Optics express. 14. 12188-95. 10.1364/OE.14.012188.

17. A. Odier, A. Durécu, J.-M. Melkonian, L. Lombard, M. Lefebvre, P. Bour don, Proc. SPIE 10083, 1008319 (2017)

18. S. Guha, J. Barnes, and L. Gonzalez, Opt. Lett. 39, 5018-5021 (2014). 
Full references (for reviewing needs only)

1. Steven J. Augst, T. Y. Fan, and Antonio Sanchez, "Coherent beam combining and phase noise measurements of ytterbium fiber amplifiers," Opt. Lett. 29, 474-476 (2004)

2. Kulp, T., Bisson, S., Bambha, R. et al., "The application of quasi-phase-matched parametric light sources to practical infrared chemical sensing systems" Appl Phys B (2002) 75: 317

3. Petter Weibring, Hans Edner, and Sune Svanberg, "Versatile mobile lidar system for environmental monitoring,"Appl. Opt. 42, 3583-3594 (2003)

4. Haris Riris, Kenji Numata, Steve Li, Stewart Wu, Anand Ramanathan, Martha Dawsey, Jianping Mao, Randolph Kawa, and James B. Abshire, "Airborne measurements of atmospheric methane column abundance using a pulsed integrated-path differential absorption lidar," Appl. Opt. 51, 8296-8305 (2012)

5. Sheng-wey Chiow, Tim Kovachy, Jason M. Hogan, and Mark A. Kasevich, "Generation of $43 \mathrm{~W}$ of quasicontinuous $780 \mathrm{~nm}$ laser light via high-efficiency, singlepass frequency doubling in periodically poled lithium niobate crystals," Opt. Lett. 37, 3861-3863 (2012)

6. Luke R. Taylor, Yan Feng, and Domenico Bonaccini Calia, "50W $C W$ visible laser source at 589nm obtained via frequency doubling of three coherently combined narrow-band Raman fibre amplifiers," Opt. Express 18, 8540-8555 (2010)

7. Gabriel Mennerat, Benoît Trophème, and Benoît Boulanger, "Experimental demonstration of five-beampumped optical parametric amplification, "Opt. Lett. 38, 3319-3321 (2013)

8. Zheng, Y.Q., Zhu, H.Y., Huang, L.X. et al., "Efficient 532 nm laser using high gray-tracking resistance KTP crystal', Laser Phys. (2010) 20: 756

9. A. Durécu, G. Canat, J. Le Gouët, L. Lombard, and P. Bourdon, "Coherent combining of SHG converters," in CLEO: 2014, OSA Technical Digest (online) (Optical Society of America, 2014), paper JTh2A.19.

10. P. Bourdon, A. Durécu, G. Canat, J. Le Gouët, D. Goular, L. Lombard, "Coherent combining of fiber-laserpumped frequency converters using all fiber electro-optic modulator for active phase control," Proc. SPIE 9344, Fiber Lasers XII: Technology, Systems, and Applications, 93441S (4 March 2015);

11. Alice Odier, Anne Durécu, Jean-Michel Melkonian, Laurent Lombard, Michel Lefebvre, and Pierre Bourdon, "Coherent combining of second-harmonic generators by active phase control of the fundamental waves," Opt. Lett. 42, 3201-3204 (2017)

12. Liwen Feng, Xiaojun Wang, and Weiwei Ke, "Coherent beam combining of optical parametric oscillators," J. Opt. Soc. Am. B 34, 991-997 (2017)
13. Arlee V. Smith, Crystal Nonlinear Optics: With SNLO Examples, AS-Photonics, 2018

14. M. M. Fejer, G. A. Magel, D. H. Jundt and R. L. Byer, "Quasi-phase-matched second harmonic generation: tuning and tolerances," in IEEE Journal of Quantum Electronics, vol. 28, no. 11, pp. 2631-2654, Nov. 1992.

15. R. Baumgartner and R. Byer, "Optical parametric amplification," in IEEE Journal of Quantum Electronics, vol. 15, no. 6, pp. 432-444, June 1979.

16. Shay, Thomas. (2007). "Theory of electronically phased coherent beam combination without a reference beam." Optics express. 14. 12188-95. 10.1364/OE.14.012188.

17. A. Odier, A. Durécu, J.-M. Melkonian, L. Lombard, M. Lefebvre, P. Bourdon, "Coherent combining of fiberlaser-pumped 3.4 um frequency converters," Proc. SPIE 10083, Fiber Lasers XIV: Technology and Systems, 1008319 (22 February 2017)

18. Shekhar Guha, Jacob O. Barnes, and Leonel P. Gonzalez, "Multiwatt-level continuous-wave midwave infrared generation using difference frequency mixing in periodically poled $\mathrm{MgO}$-doped lithium niobate," Opt. Lett. 39, 5018-5021 (2014) 